\title{
Dynamic Modeling of Radar System Failure Diagnosis Based on the UML Technology \\ Yu Zou ${ }^{1, a}$, Kai Li ${ }^{2, b}$ and Wenjing Yang ${ }^{3, c}$
}

${ }^{1}$ China Satellite Maritime Measurement and Control Department, Jiangyin, China

${ }^{2,3}$ China Satellite Maritime Tracking and Control Department, Jiangyin, China

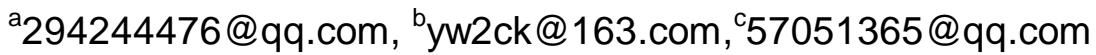

Keywords: UML; Radar system; Fault diagnosis; Dynamic modeling; Research

\begin{abstract}
This article Uses the more popular now unified modeling language UML for a type of school shooting Radar fault diagnosis expert system software to set up a general dynamic model, the system software design of the systematic, modular and thus greatly improve the generality, the standardization and the reliability of the system software.
\end{abstract}

\section{Introduction}

Radar fault diagnosis and isolation of expert system software is a model school shot one of the important parts of Radar, it troubleshooting, repair and maintenance of the Radar system has played a crucial role, at the same time, it also contains a large amount of information about Radar and subsystem information, expert diagnostic information and the using method of the system software, convenient for system administrators and test engineer on Radar system query and query to the method of the system software USES, greatly improve the versatility and convenience of the system. Before the design of Radar fault diagnosis system since there is no uniform design idea, design scheme and Radar model and design is different, so there are a lot of Radar fault diagnosis system software, each in the design of system software structure, software interface, system function, operation steps, etc, which can lead to poor universality of the system software. Plus large software development cycle is long, many characteristics such as participating in the development, there is no unified and clear design document for your reference, for the various stages of the same set of system software development, the developer is not easy to communicate, exchange and inheritance. This leads to an increase in system development costs and is not easily maintained and upgraded. This article will use the unified modeling language UML, for the institute to develop a type of the frame structure of shoot Radar general software system design, make the testing software system standardization and visualization.

\section{Fundamental Principles}

Unified Modeling Language UML Profiles. Unified modeling language (UML) it defines a standard language used to simplify the system model and graphic symbol, can demand, the flow chart of activities, the function of the system structure design and system development and the establishment of the model, such as products of the software system for detailed (Specifying), visualization, visualizing, ConstruCting (ConstruCting), document (doeumenting). The purpose of using UML modeling and visualization is the ability to make through graphical representation of the developers to capture system related important details, such as the needs of the project analysis can be understand, the solution is to get effective implementation, etc., as a kind of support object-oriented analysis and design modeling language, UML can express not only the basic concept of function block, also provides several ways to represent the complicated relationship between basic function block, the relationship between the implication of the rich in the UML chart.

The Composition of the Expert System of Radar Matrix Diagnostics. The Radar fault diagnosis expert system used in this article is one of the important components of a certain type of Radar product, 
which can be based on the experience of Radar experts. The data is used to guide the tester to the fault diagnosis and location of the Radar. The Radar fault diagnosis system is mainly composed of two parts: hardware part and software part, the software part consists of fault diagnosis test system and expert experience, the test system provides three test strategy: BITE diagnosis strategy, phenomenon of fault diagnosis and systemic examination. Tester can choose these strategies under the guidance of test system to complete fault diagnosis of Radar, positioning, with the help of the fault diagnosis expert system, the maintenance personnel can don't need to know a lot of Radar in terms of professional knowledge can be smooth, quick and accurate fault Radar maintenance work, and can use the software to realize the Radar's regular maintenance work. Hardware part is composed of analog part and mixed part, mainly through expert experience diagnosis subsystem process found suspicious replaceable units (LRU), will be suspect LRU get corresponding test bed, realize fault location.

\section{The Dynamic Modeling of Radar Fault Diagnosis System}

The UML status view mainly describes the behavior of the system, which is to describe the timing and interaction of the set of models that can be executed or executed. The main USES are status diagrams, activity diagrams, sequence diagrams, and collaboration diagrams. In object-oriented technology, interactions between objects are accomplished through the transfer of messages between objects. Objects work together through the messaging of each other and change their state in the lifecycle of their life. This mechanism is adopted in the four dynamic models of UML. Here we use some of the elements on the state diagram to model the state diagram of some more complex state or behavior in the troubleshooting system software.

The State Diagram of the Troubleshooting System is Implemented. Fault diagnosis state diagram represents a user from the login to complete the change of state to fault diagnosis of the whole process, allows users to clearly understand the software when performing fault diagnosis of the workflow, state of the whole process is: the user first login request, according to login information decision system of the state, after the success of the login into the diagnostic process selection interface, to obtain correct diagnosis strategy, and the diagnosis strategy under the premise of existence, the user can according to the diagnostic strategy prompts fault diagnosis of Radar. When the diagnostic process is complete, the security exit is the entire state of the diagnosis.

The Process of Diagnosis System Activity is Implemented. Radar fault diagnosis system is the core problem is the process of fault diagnosis, this software in the process of open the test project, accompanied by the instrument online test engineering and transferred to the occurrence of two activities, including the online test is the connectivity test instrument, Radar subsystem under test (UUT) piece test only in the case of online test instrument can correctly reflect the state of the UUT, so in the open engineering as well as in the linear test for testing instruments; The input test engineering information is information about Radar and subsystem, system software USES information. Expert experience process information and UUT test load testing software, into the expert experience in the process of the fault diagnosis process, you can query all kinds of information at any time, diagnose can continue to process the query, which greatly increases the flexibility and reliability of the system software.

The system software provides two ways to test the Radar subsystem's UIJT test: one is in the expert experience process, and the test is dynamic based on the need to be tested; The other way is to go to the Radar subsystem and then click directly to test. Figure 1 is an activity diagram that directly tests the UUT. Before testing UUT directly, you can test the instrument online and test it as needed, which will increase the effectiveness of the test process. Of course, you can also go directly to the test interface, connect UUT with the test instrument, and then start testing. Pay attention to in the process of test, accompanied by two activities simultaneously, one is send commands to the device requests, and obtain real-time instrument read data is returned to the testing interface to save; The other is a test process that is designed in the development system that is designed according to the internal structure and working principle of the UUT. Problems when, in the event of test failures may be because the cause of the instrument, also may be the cause of the test method, this is where the instruments and test methods for 
judgment, so as to find out the problem. Note: the UUT test activity diagram is the UUT itself under the premise of normal design, if the UUT test is no guarantee that the normal, the test results to go, just think UUT fault.

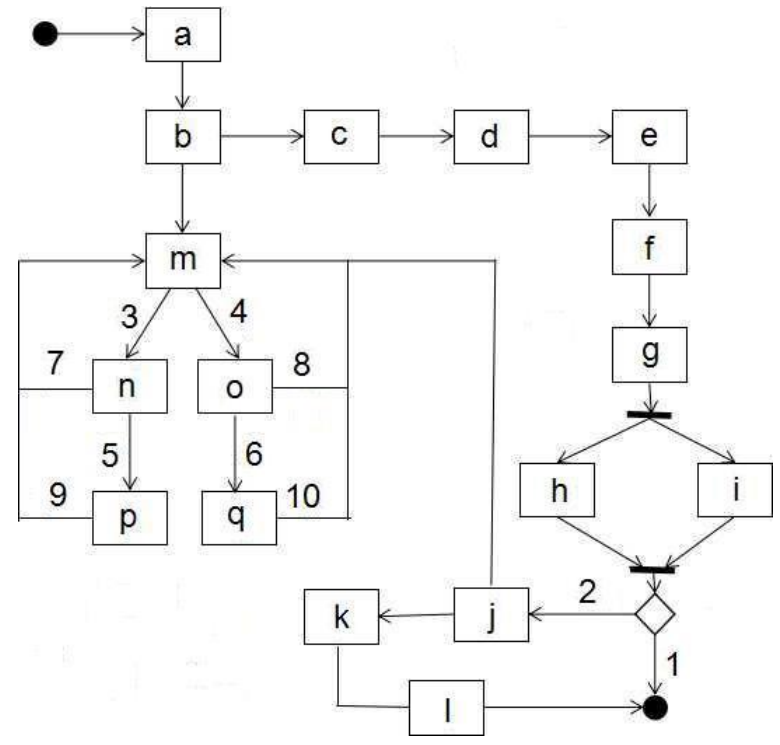

Figure1 UUT test activity diagram

a. subsystem diagnostic strategy ;b. subsystem interface ;c. choose the UUT;d. Click "test" ;e.Enter the test main screen; f.See the prompt for information; g. Enter the diagnostic process; h. Call the test instrument;i.I invoke the test process;j. The test doesn't pass;k. Check the test method; $1 . U$ UT fault;m. instrument testing;n. online test;o. online test;p. self test;q. Self test;1.Pass;2.Not pass;3.The simulation;4.combination;5.Online;6.Not online; 7.Not online; 8.Not online; 9.Not pass;10.Not pass.

The UTT Fault Diagnosis Sequence Diagram is Implemented. The UUT fault diagnosis sequence diagram is shown in figure 2. Fault diagnosis personnel on the premise of not need expertise to do guidance, can roughly judge a subsystem of a certain Radar board may malfunction, can directly enter the subsystem test, and don't need to find the board step by step from the diagnostic process, which greatly increased the flexibility of the system software, the fault diagnosis personnel first choose corresponding diagnosis method to get into the subsystem interface subsystem, in the subsystem interface tester can choose any UUT, right-click test, after entering the UUT test interface, diagnosis of personnel should be according to the UUT diagnostic message connect the UUT and testing instruments, under the condition of the connection are correct, choose the UUT diagnosis process test, the test data will be saved in the form of statements in diagnostic statements, so the test data will eventually be returned to the testers in the form of statements, each test result will be promptly returned to the diagnosis of personnel, personnel so that the fault diagnosis of UUT has a timely, accurate understanding and facilitate system maintenance and UUT maintenance. 


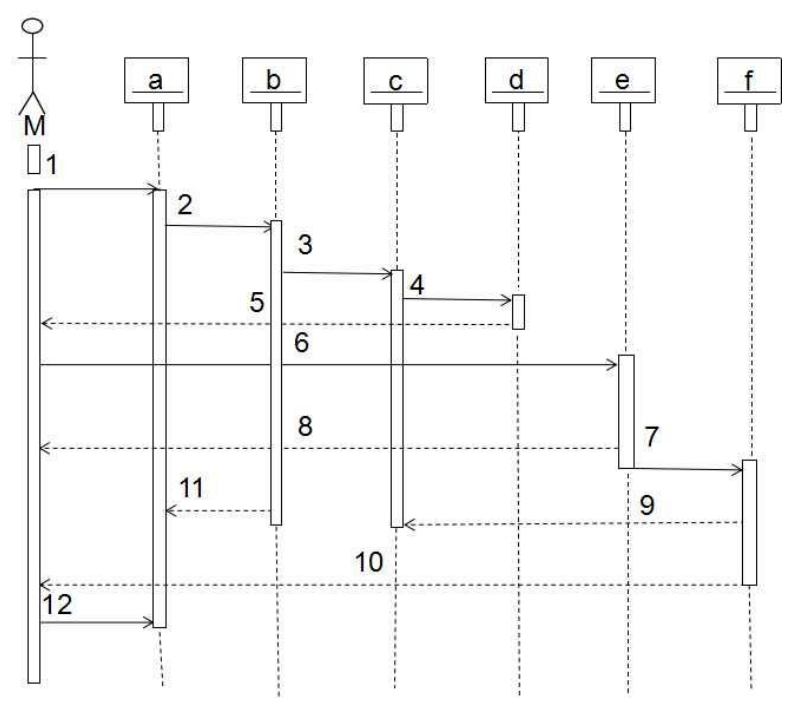

Figure 2 UUT fault diagnosis sequence diagram

M: Troubleshooer;a. radar interface;b. subsystem interface; c.U UT test interface;d. diagnostic information;e. diagnostic procedure $f$. diagnostic reports;1.Open the diagnostic method; 2.Enter the subsystem interface; 3.Click the UUT test; 4.Find the test prompt information;5.Getting the UUT test information; 6.Enter the diagnostic process interface; 7.Generate test reports; 8.The UTT diagnosis returned; 9.A UUT diagnosis is over; 10.Return the test report information;11. The diagnosis of a subsystem is over; 12 . Save the test report

Collaboration Diagram for Troubleshooting System. Collaboration diagram is used to describe the behavior of the system is that if the system components of the collaboration diagram, collaboration diagram modeling elements are included in the object (including participants instance, many objects, active objects, etc.), news, chains, etc. The collaboration diagram USES a link to connect the object, and the message appears next to the chain, with multiple messages on a chain. Sequence diagrams and collaboration diagrams are both interactive diagrams that describe dynamic relationships between objects in the system. The two can be exchanged, but the emphasis is different. The sequence diagram emphasizes the chronological order of the message, while the collaboration diagram emphasizes the organization of the objects participating in the interaction. On the modeling elements used in the two diagrams, they also have their own characteristics. In the sequence diagram, there is the object lifeline and the control focus, not in the collaboration diagram; There is a path in the collaboration diagram, and the message in the collaboration diagram must have a message sequence number, but there are no two characteristics in the sequence diagram. Compared to the collaboration diagram, the sequence diagram is the presentation algorithm. The life cycle of an object. The object of the specific multithreaded characteristics is relatively easy, but it is harder to represent concurrent control flows. Sequence diagrams and collaboration diagrams are semantically equivalent, and they can be exchanged between them, but they cannot be substituted for each other. The sequence diagram can represent information that some collaboration diagrams cannot represent, and also the collaboration diagram can represent information that is not represented in some sequence diagram. In view of the many similarities between the collaboration diagram and the sequence diagram, the collaborative diagram modeling of Radar fault diagnosis system software is not established in this paper.

\section{Conclusion}

Unified modeling language UML is a kind of semantic rich visual modeling language, it supports the whole process starting from the requirements analysis of software development, help to establish a clear and intuitive system model, is suitable for the complex systems in many areas, is a highly efficient and flexible incremental iterative development process, it can be effective to the project planning and management, reduce risk, improve quality, reduce the complexity of software development, improve the 
efficiency of software development. Use modeling tools and software development to adapt to modern social requirements for software development. This article USES the more popular now unified modeling language UML to design the system dynamic model, and implements the Radar, the framework of fault diagnosis expert system software has been successfully developed and Radar target in a model school passed the verification.

\section{References}

[1] (U.S.) Schmoller (Schmuller, J.) forward. UML-based, case studies and applications [M]. 3rd ed. Li-hu, ZHAO just translated. Beijing: People's Posts \&amp; Telecom Press, 2004. [3 ] Ye Bin. software development in the use-case analysis techniques [J]. microcomputer development, 2004, 14 (9) :118-121.

[2] Zhang Zhibin, Chen Sheng. Based on the UML-fire response alarm systems development modeling [J]. Computer Engineering and Science, 2005,27 (1):77-79.

[3] XU Bao-wen, ZHOU Yu-Ming, Lu Hong-Min. UML and software modeling [M]. Beijing: Tsinghua University Press, 2006, (6).

[4] Joseph Schmuller a, Li-hu, ZHAO just translated. UML-based, case studies and applications [M]. Beijing: People's Posts \& Telecom Press, 2007, (1).

[5] [America] Michael Blaha, James Rumbaugh forward. Object-Oriented Modeling and Design with UML [M]. Beijing: People's Posts \& Telecom Press, 2006, (7).

[6] Xu Tianyu compiled. E-commerce System Planning and Design [M]. Beijing: Tsinghua University Press, 2006, (5).

[7] Shi Ji-min. Software Engineering [M]. Beijing: Higher Education Press, 2002. [8] Shao-Feng Wang. Object-oriented UML Tutorial [M]. Beijing: Tsinghua University Press, 2004.

[8] Zheng Li, Dong Yuan. C language programming [M]. Beijing: Tsinghua University Press, 2004. 